\title{
TIME-FREQUENCY SYNTHESIS OF STATISTICALLY MATCHED WEYL-HEISENBERG PROTOTYPE SIGNALS*
}

\author{
W. Kozek, H.G. Feichtinger and T. Strohmer \\ Numerical Harmonic Analysis Group \\ Department of Mathematics, University Vienna \\ Strudlhofgasse 4, A-1090 Vienna, Austria \\ (kozek@tyche.mat.univie.ac.at)
}

\begin{abstract}
The use of a family of time-frequency shifted versions of a prototype signal, namely Weyl-Heisenberg (WH) sets, is a common feature of various concepts in signal analysis, processing and digital communication. We discuss the matching of WH prototype signals to important nonstationary environments. The statistical window optimization for the STFT/Gabor expansion of nonstationary processes is shown to be formally equivalent to optimal signal design for narrowband WSSUS channels. For the case of underspread channels/processes we derive approximate solutions to the optimization problem.
\end{abstract}

\section{INTRODUCTION}

Eigenfunctions and the associated signal expansions provide the optimum solution to a wide variety of signal processing problems. An obvious example is the transmission over a linear channel where the transmission pulses should be eigensignals of the channel. Another example is the Karhunen-Loeve transform that diagonalizes the covariance operator of a nonstationary process and thus establishes the theoretically optimum transform for minimum mean-squared error filtering and source coding. Nevertheless, exact (and usually unstructured) eigenexpansions are seldom used in practice, rather one uses highly structured signal bases. There are two main, pragmatical reasons for doing so: (i) the a priori knowledge on a linear operator is essentially incomplete (as e.g. in mobile communication) such that it does not come to the point of solving the eigenvalue problem, and (ii) the numerical expense for the use of a general (unstructured) linear transform is too high.

A classical version of a structured set of functions is obtained by time-frequency shifting of a prototype function $\mathrm{g}(\mathbf{t})$,

$$
g^{(\tau, \nu)}(t) \stackrel{\text { def }}{=} g(t-\tau) e^{j 2 \pi \nu t}
$$

The associated signal transforms are the short-time Fourier transform (STFT) $[1,2]$

$$
\operatorname{STFT}_{x}^{(g)}(t, f) \stackrel{\text { def }}{=}\left\langle x, g^{(t, f)}\right\rangle
$$

and its discretized version, the Gabor expansion, whose coefficients may be written as $[1,3,4,5]$

$$
G_{x}^{(g)}(m, n)=S T F T_{x}^{(g)}(m T, n F),
$$

where $g(t)$ is an appropriately defined Gabor analysis window and $T F<1$ assures completeness in $L_{2}$-sense.

In digital communication (in particular time/frequencydivision multiplex systems) one uses discrete WH sets $\left\{g^{(m T, n F)}(t)\right\}$ of a transmission pulse. Here, one always has $T F>1$ since linear independence is compelling and completeness is not at all important [6].

\footnotetext{
*Funding by grant 4913 of the Jubiläumsfonds der
} Österreichischen Nationalbank and FWF grant S7001-MAT.
In the context of the WH group the appropriate tool for operator representation is the generalized spreading function [7],

$$
S_{H}^{(\alpha)}(\tau, \nu) \stackrel{\text { def }}{=} \int_{t} h\left(t+\left(\frac{1}{2}-\alpha\right) \tau, t-\left(\frac{1}{2}+\alpha\right) \tau\right) e^{-j 2 \pi \nu t} d t
$$

where $h(.,$.$) is the kernel of a Hilbert-Schmidt (HS) opera-$ tor $\mathbf{H}$. The real-valued parameter $\alpha$ reflects the nonuniqueness in the definition of a WH time-frequency shift operator. However, most of our results are $\alpha$-invariant since the magnitude of $S_{H}^{(\alpha)}(\tau, \nu)$ does not depend on $\alpha$. The generalized spreading function appears in various contexts. Since we shall utilize these crossconnections we give a brief survey:

- When the operator $\mathbf{H}$ corresponds to a linear, timevarying system, then $S_{H}^{(1 / 2)}(\tau, \nu)$ is called the delayDoppler spread function [8];

- In applied mathematics [9] the generalized spreading function appears as the symplectic Fourier transform of the generalized Weyl symbol $L_{H}^{(\alpha)}(t, f)$, i.e., the Weyl symbol in particular for $\alpha=0$ and the Kohn-Nirenberg symbol, or, equivalently, time-varying transfer function for $\alpha=1 / 2[7]$ :

$$
S_{H}^{(\alpha)}(\tau, \nu)=\int_{t} \int_{f} L_{H}^{(\alpha)}(t, f) e^{-j 2 \pi(\nu t-\tau f)} d t d f .
$$

- For rank-one projections, $g \otimes g, S_{g \otimes g}(\tau, \nu)$ is wellknown as generalized radar ambiguity function of the signal $g(t)[1,10,11,12]$.

- For a correlation operator $\mathbf{R}_{x}$ whose kernel is the autocorrelation function of a nonstationary process, $\left(\mathbf{R}_{x}\right)\left(t, t^{\prime}\right)=E\left\{x(t) x^{*}\left(t^{\prime}\right)\right\}$, the spreading function can be interpreted as stochastic time-frequency correlation function of the process [13].

\section{MATCHED CONTINUOUS EXPANSIONS}

The continuous expansion set $\left\{g^{(t, f)}\right\}$ is highly linear dependent. In a recent work [2] it has been shown that notwithstanding the high linear dependence of the set it makes sense to study its diagonalizing properties in the sense of a continuous off-diagonal norm. Based on a continuous 4D kernel,

$$
H_{g}\left(t, f, t^{\prime}, f^{\prime}\right)=\left\langle\mathbf{H} g^{\left(t^{\prime}, f^{\prime}\right)}, g^{(t, f)}\right\rangle,
$$

one can formulate a prototype optimization criterion subject to $\|g\|^{2}=1$ as follows:

$$
g_{o p t, C}=\arg \min _{\substack{g \\ t, t^{\prime}, f, f^{\prime}}}\left|H_{g}\left(t, f, t^{\prime}, f^{\prime}\right)\right|^{2} W\left(t-t^{\prime}, f-f^{\prime}\right) d t d t^{\prime} d f d f^{\prime}
$$


where $W(t, f)$ is a radially nondecreasing weight function with $W(0,0)=0$ and $0<W(t, f)<1$ that penalizes the off-diagonal spread of $H_{g}\left(t, f, t^{\prime}, f^{\prime}\right)$.

For a given $H S$ operator $\mathbf{H}$ one can find a numerically tractable reformulation of the optimization problem in terms of the smoothed spreading function of the operator and the ambiguity function of the prototype signal:

$$
g_{\text {opt }, C}=\arg \max _{g}\left\langle\left|S_{H}\right|^{2} * *(1-W),\left|S_{g \otimes g}\right|^{2}\right\rangle, \quad\|g\|^{2}=1,
$$

where ** denotes the convolution of $2 \mathrm{D}$ functions.

An existing application of the matched continuous expansion is discussed in [2]. When the HS operator at hand is a correlation operator the kernel defined by (4) carries over to the correlation of the STFT coefficients

$$
\left\langle\mathbf{R}_{x} g^{\left(t^{\prime}, f^{\prime}\right)}, g^{(t, f)}\right\rangle=\mathrm{E}\left\{S T F T_{x}^{(g)}(t, f) S T F T_{x}^{(g) *}\left(t^{\prime}, f^{\prime}\right)\right\},
$$

and the prototype optimization gives the STFT analysis window with optimally uncorrelated coefficients.

\section{MATCHED DISCRETE EXPANSIONS}

The matching of a discrete WH set $\left\{g^{(m T, n F)}\right\}$ to a HS operator has been recently studied for the specific case of a correlation operator [5]. This derivation was based on specific assumptions on $\mathbf{H}$ and $T, F$. We here discuss a more general setup consistent with [5].

Consider a discrete WH set with elements defined by

$$
g^{(m T, n F)}(t)=g\left(t-m T+\tau_{0}\right) e^{j 2 \pi\left(n F-\nu_{0}\right) t}
$$

where $\tau_{0} \in[0, T], \nu_{0} \in[0, F]$ denote the sampling phase of the time-frequency grid. The explicit consideration of the sampling phase is a key point in the following derivation.

The action of the operator $\mathbf{H}$ on the WH set determines a 4D discrete kernel:

$$
H_{g}\left(m, n, m^{\prime}, n^{\prime}\right) \stackrel{\text { def }}{=}\left\langle\mathbf{H} g^{\left(m^{\prime} T, n^{\prime} F\right)}, g^{(m T, n F)}\right\rangle .
$$

The discrete WH set is characterized by the prototype function $g(t)$, the grid constants $T, F$ and the sampling phases $\tau_{0}, \nu_{0}$. The matching of the expansion set in the sense of optimum diagonalization of $\mathbf{H}$ can be written as

$$
\left(g, T, F, \tau_{0}, \nu_{0}\right)_{o p t}=\arg \min _{\left(g, T, F, \tau_{0}, \nu_{0}\right)} M_{W}^{(g)}\left(T, F, \tau_{0}, \nu_{0}\right),
$$

subject to $\|g\|^{2}=1$ and $T F=k$, where $k$ is an application dependent constant and $M_{W}^{(g)}\left(T, F, \tau_{0}, \nu_{0}\right)$ denotes an offdiagonal norm:

$M_{W}^{(g)}\left(T, F, \tau_{0}, \nu_{0}\right)=\sum_{m, m^{\prime}, n, n^{\prime}}\left|H_{g}\left(m, n, m^{\prime}, n^{\prime}\right)\right|^{2} W\left(m-m^{\prime}, n-n^{\prime}\right)$,

where $W(m, n)$ is a nonnegative off-diagonal measure $(W(0,0)=0$ and radially nondecreasing).

Random Phase Approach. The general optimization problem (7) is too complicated for a numerically tractable solution. Moreover, in most of the practical applications there is no possibility to adapt the sampling phase of the WH set. Hence, we eliminate the sampling phase dependence via randomization, where the time and frequency sampling phases are uniformly distributed random variables with probability density functions

$$
p_{\tau_{0}}(\xi)=\frac{1}{T} \chi_{[0, T]}(\xi) \quad \text { and } \quad p_{\nu_{0}}(\xi)=\frac{1}{F} \chi_{[0, F]}(\xi),
$$

where $\chi_{[0, T]}(\xi)$ is the indicator function of the interval $[0, T]$. The expectation of the discrete off-diagonal norm w.r.t. the uniformly distributed random sampling phase leads to [14]:

$$
\begin{aligned}
E_{\tau_{0}, \nu_{0}} & \left\{M_{W}^{(g)}\left(T, F, \tau_{0}, \nu_{0}\right)\right\}=\frac{1}{T F} \sum_{\Delta m} \sum_{\Delta n} W(\Delta m, \Delta n) \\
\cdot & \int_{\tau} \int_{\nu}\left|S_{H}(\tau, \nu)\right|^{2}\left|S_{g \otimes g}(\tau-\Delta m T, \nu-\Delta n F)\right|^{2} d \tau d \nu,
\end{aligned}
$$

For given grid constants, the window optimization problem subject to $\|g\|^{2}=1$ is thus structurally equivalent to (5):

$$
g_{\text {opt }, D}=\arg \max _{g}\left\langle 1-\left|\widehat{S}_{H}^{(T, F)}\right|^{2},\left|S_{g \otimes g}\right|^{2}\right\rangle,
$$

where $\widehat{S}_{H}^{(T, F)}(\tau, \nu)$ is a weighted-periodized version of the operator's magnitude-squared spreading function:

$\left|\widehat{S}_{H}^{(T, F)}(\tau, \nu)\right|^{2} \stackrel{\text { def }}{=} \sum_{m} \sum_{n} W(m, n)\left|S_{H}(\tau-m T, \nu-n F)\right|^{2}$.

Matched Grid for Underspread Operators. In the context of approximate operator diagonalization via $\mathrm{WH}$ sets operators with restricted spreading function play a distinguished role. We characterize the spreading constraint by a $0 / 1-v a l u e d$ indicator function $\chi_{H}(\tau, \nu)$ :

$$
S_{H}^{(\alpha)}(\tau, \nu)=S_{H}^{(\alpha)}(\tau, \nu) \chi_{H}(\tau, \nu) .
$$

In order to obtain closed form analytical results we restrict the discussion to a rectangular shape of $\chi_{H}(\tau, \nu)$ characterized by $\left[-\tau_{\max }, \tau_{\max }\right] \times\left[-\nu_{\max }, \nu_{\max }\right]$. When the area of this centered rectangle is smaller than one we call the operator underspread and in the converse case overspread [14]. For such underspread operators the generalized Weyl symbol $L_{H}^{(\alpha)}(t, f)$ is a $2 D$ lowpass function that is uniquely characterized by its samples on a rectangular grid with $T=\frac{1}{2 \nu_{\max }}$ and $F=\frac{1}{2 \tau_{\max }}$. This leads to a discrete WH expansion of the operator [14]

$$
\mathbf{H}=\sum_{m} \sum_{n} L_{H}^{(\alpha)}(m T, n F) \mathbf{S}^{(m T, n F)} \mathbf{P}(\alpha) \mathbf{S}^{(m T, n F)+},
$$

where the prototype operator $\mathbf{P}(\alpha)$ is given by $S_{P(\alpha)}^{(\alpha)}(\tau, \nu)=\chi_{H}(\tau, \nu), \mathbf{S}^{(\tau, \nu)}$ denotes the WH timefrequency shifting operator and the grid ratio is

$$
\frac{T}{F}=\frac{\tau_{\max }}{\nu_{\max }} \text {. }
$$

Regarding the problem of matching a discrete WH set to an underspread operator, we have typically $T F<1$ while the critical grid for the symbol sampling (9) leads to $T F>1$. However, we suggest to use the matched grid ratio (10) for any value of the product $T F$ underlying a WH set. We have no strict proof for optimality, but apart from the symbol sampling argument there are two other lines of reasoning that all lead to (10).

Via Frame Theory. For elliptical symmetry of $\chi_{H}(\tau, \nu)$ the optimum prototype signal for continuous expansions is a Gaussian pulse with matched scale (to be discussed in Sec. 5). Both the theoretical result in [4] and numerical results in [3] show that for the matched Gaussian, the matched grid ratio (10) leads to the tightest WH frame for $T F<1$.

Via Residual Off-Diagonal Norm. For underspread operators the residual off-diagonal norm will be dominated by the nearest side-diagonals $|\Delta m|=1,|\Delta n|=1$ such that it suffices to consider a $\widehat{S}_{H}^{(T, F)}(\tau, \nu)$ with $W(m, n)=0$ 
whenever $m \neq 0$ or $n \neq O$. For a $\chi_{H}(\tau, \nu)$ with rectangular shape the centered gap left by $\widehat{S}_{H}^{(T, F)}(\tau, \nu)$ is an approximate measure for the residual off-diagonal norm since $S_{g_{o p t} \otimes g_{o p t}}(\tau, \nu)$ will be well concentrated about the origin. Optimizing the area of the gap rectangle left by $\widetilde{S}_{H}^{(T, F)}(\tau, \nu)$ should minimize the residual off-diagonal norm. This optimization leads again to the matched grid ratio (10).

\section{SIGNAL DESIGN FOR WSSUS CHANNELS}

We now show that the prototype optimization criteria discussed in the foregoing section are applicable to the optimum signal design in the context of communication over time-varying channels.

WSSUS. Wide-Sense Stationary Uncorrelated Scattering (WSSUS) is a statistical model for time-varying communication channels, particularly for the mobile radio channel $[8,7]$. In the WSSUS model, $\mathbf{H}$ is a stochastic linear timevarying system characterized by a zero-mean generalized spreading function with correlation

$$
\mathrm{E}\left\{S_{H}^{(\alpha)}(\tau, \nu) S_{H}^{(\alpha) *}\left(\tau^{\prime}, \nu^{\prime}\right)\right\}=C_{H}(\tau, \nu) \delta\left(\tau-\tau^{\prime}\right) \delta\left(\nu-\nu^{\prime}\right)
$$

where $C_{H}(\tau, \nu)$ is the scattering function of the channel. The a priori knowledge of the scattering function is clearly insufficient to determine eigensignals. Moreover, two individual realizations may not have any common eigensignal. Hence, it does not make sense to ask for eigensignals of a stochastic system.

Minimum Expected Distortion. Notwithstanding the nonexistence of eigensignals one may still ask for an optimum signal which comes closest to an eigensignal in the sense of minimum expected orthogonal distortion. The orthogonal distortion of an unit norm input signal $g(t)$ is given by

$$
\left\|\epsilon_{H, g}\right\|^{2}=\|\mathbf{H} g\|^{2}-|\langle\mathbf{H} g, g\rangle|^{2}, \quad\|g\|=1 .
$$

The minimization of the expectation $\left\|\epsilon_{H, g}\right\|^{2}$ over the WSSUS ensemble leads to the following optimization problem subject to $\|g\|=1$

$$
g_{o p t}=\arg \min _{g} \mathrm{E}\left\{\left\|\epsilon_{H, g}\right\|^{2}\right\}=\arg \max _{g}\left\langle C_{H},\left|S_{g \otimes g}\right|^{2}\right\rangle,
$$

which is equivalent to the continuous WH matching criterion (5) when one replaces the magnitude squared spreading function $\left|S_{H}(\tau, \nu)\right|^{2}$ by the channel's scattering function $C_{H}(\tau, \nu)$.

Minimum Expected Interference. The above discussed optimum single pulse may be of interest as a test signal, but for digital communication one has to take into account interference from time-frequency neighbouring pulses. We here consider a time-frequency division setup where the input signal is a weighted linear combination of a WH set based on a transmission pulse $g(t)$ :

$$
x(t)=\sum_{m} \sum_{n} p(m, n) g^{(m T, n F)}(t)
$$

One may identify $T$ as the symbol rate and $F$ as the channel separation. However, we treat cross and self interference in a common manner such that our results are independent of the actual rule for channel access.

We furthermore assume uncorrelated pulse amplitudes with normalized power:

$$
\mathrm{E}_{p}\left\{p(m, n) p^{*}\left(m^{\prime}, n^{\prime}\right)\right\}=\delta_{m m^{\prime}} \delta_{n n^{\prime}},
$$

where $\delta_{m m^{\prime}}$ is the Kronecker symbol $\left(\delta_{m m^{\prime}}=1\right.$ for $m=$ $m^{\prime}$ else zero). A matched filter receiver for the symbol associated to $g^{(m T, n F)}(t)$ evaluates an inner product of $g^{(m T, n F)}(t)$ and the channel's output signal $(\mathbf{H} x)(t)$ (we assume noise free observation):

$$
\hat{p}(m, n)=\left\langle\mathbf{H} x, g^{(m T, n F)}\right\rangle=\sum_{m^{\prime}, n^{\prime}} p\left(m^{\prime}, n^{\prime}\right) H_{g}\left(m, n, m^{\prime}, n^{\prime}\right),
$$

where we have used the kernel defined in (6). Due to the distortion caused by the channel the matched filter output contains undesirable contributions from other pulses. The optimum transmission pulse minimizes the total expected energy of all interfering distorted pulses:

$$
\begin{aligned}
g_{\text {opt }}= & \arg \min _{g} \mathrm{E}_{H, p}\left\{\mid \sum_{m^{\prime}, n^{\prime}} p\left(m^{\prime}, n^{\prime}\right)\right. \\
& \left.\left.\cdot H_{g}\left(m, n, m^{\prime}, n^{\prime}\right) W\left(m-m^{\prime}, n-n^{\prime}\right)\right|^{2}\right\},
\end{aligned}
$$

where the expectation is over the WSSUS channel and the amplitude ensemble, and the weight function $W(m, n)$ selects contributions from pulses with different TF localization:

$$
W\left(m-m^{\prime}, n-n^{\prime}\right) \stackrel{\text { def }}{=} 1-\delta_{n n^{\prime}} \delta_{m m^{\prime}} .
$$

In order to compute the expectation in (12) we note that $\mathrm{E}_{p}\left\{\left|\sum_{m^{\prime}} \sum_{n} p\left(m^{\prime}, n^{\prime}\right) H_{g}\left(m, n, m^{\prime}, n^{\prime}\right)\right|^{2}\right\}=$ $\sum_{m^{\prime}} \sum_{n^{\prime}}\left|H_{g}\left(m, n, m^{\prime}, n^{\prime}\right)\right|^{2}$ and, since in general

$$
\mathrm{E}_{H}\left\{\left|\left\langle S_{H}, S_{x \otimes y}\right\rangle\right|^{2}\right\}=\left\langle C_{H},\left|S_{x \otimes y}\right|^{2}\right\rangle
$$

such that specifically

$$
\begin{aligned}
\mathrm{E}_{H}\left\{\left|H_{g}\left(m, n, m^{\prime}, n^{\prime}\right)\right|^{2}\right\}=\int_{\tau} \int_{\nu} C_{H}(\tau, \nu) \\
\quad \cdot\left|S_{g \otimes g}\left(\tau-\left(m-m^{\prime}\right) T, \nu-\left(n-n^{\prime}\right) F\right)\right|^{2} d \tau d \nu .
\end{aligned}
$$

Without loss of generality we consider the pulse with $m=$ $0, n=0$ and combining the above results we have

$$
\mathrm{E}_{H}\left\{\sum_{m^{\prime}, n^{\prime}}\left|\left\langle\mathbf{H}_{g}^{\left(m^{\prime} T, n^{\prime} F\right)}, g\right\rangle\right|^{2}\right\}=\left\langle 1-\widehat{C}_{H}^{(T, F)},\left|S_{g \otimes g}\right|^{2}\right\rangle,
$$

with $\widehat{C}_{H}^{(T, F)}(\tau, \nu) \stackrel{\text { def }}{=} \sum_{m} \sum_{n} W(m, n) C_{H}(\tau-m T, \nu-$ $n F)$. Hence, the optimization of the transmission pulse according to (12) is equivalent to the matching criterion for discrete WH expansions when one replaces $\left|\widehat{S}_{H}^{(T, F)}(\tau, \nu)\right|^{2}$ by $\widehat{C}_{H}^{(T, F)}(\tau, \nu)$ in $(8)$.

\section{APPROXIMATE MATCHING}

Via Symbolic Calculus. As discussed in Sec. 3, the generalized Weyl symbol provides a discrete WH expansion of underspread operators (9). The interpretation of the generalized Weyl symbol suggests to view (9) as a time frequency-parametrized spectral decomposition of the operator [14]. However, the prototype operator $\mathbf{P}(\alpha)$ is not rank-one, it is natural to define the optimum prototype signal via optimum rank-one approximation of the prototype operator in a HS sense:

$$
\tilde{g}_{o p t}^{(\alpha)}=\arg \min _{g}\|\mathbf{P}(\alpha)-g \otimes g\|_{H S},
$$


subject to $\|g\|=1$. It is easy to show that this is equivalent to

$$
\tilde{g}_{o p t}^{(\alpha)}=\arg \min _{g}\left\langle\chi_{H}, S_{g \otimes g}^{(\alpha)}\right\rangle, \quad\|g\|=1 .
$$

This is just the classical setup for least-squares synthesis of radar ambiguity functions $[11,12]$. Note that (13) basically depends on $\alpha$ while the "exact" matching criteria (5), (8) are $\alpha$-independent:

$$
g_{o p t}=\arg \min _{g}\left\langle T_{H},\left|S_{g \otimes g}^{(\alpha)}\right|^{2}\right\rangle, \quad\|g\|=1,
$$

where $T_{H}(\tau, \nu)$ stands for either the magnitude squared spreading function or the scattering function. In the case of an incomplete a priori knowledge in the form of $\chi_{H}(\tau, \nu)$ it is natural to use $\chi_{H}(\tau, \nu)$ instead of $T_{H}(\tau, \nu)$. The numerical solution of $(13)$ essentially reduces to a partial eigenvalue problem which is less expensive compared to the iterative solution of (14). We emphasize that with decreasing spread the $\alpha$-variance of (13) gets more and more negligible.

Strongly Underspread Operators. For many applications a rough matching of the prototype signal may be sufficient. In order to obtain a simple, approximate window matching rule we assume strongly underspread operators, i.e.,

$$
\tau_{\max } \nu_{\max } \ll 1
$$

with either rectangular

$$
\chi_{H}(\tau, \nu)=\chi_{\left[-\tau_{\max }, \tau_{\max }\right]}(\tau) \chi_{\left[-\nu_{\max }, \nu_{\max }\right]}(\nu)
$$

or elliptical shape

$$
\chi_{H}(\tau, \nu)=\left\{\begin{array}{ll}
1, & \left(\frac{\tau}{\tau_{\max }}\right)^{2}+\left(\frac{\nu}{\nu_{\max }}\right)^{2} \geq 1 \\
0, & \text { else }
\end{array},\right.
$$

and we assume that $S_{H}(\tau, \nu) \approx \chi_{H}(\tau, \nu)$. Due to (15) one can replace the ambiguity function a of (real-valued) window by its Taylor approximation $[11,10]$ :

$$
S_{g \otimes g}(\tau, \nu) \approx 1-\frac{1}{2 \pi}\left(F_{g}^{2} \tau^{2}+T_{g}^{2} \nu^{2}\right)
$$

where $T_{g}^{2}$ and $F_{g}^{2}$ are the temporal and spectral moments of order two of the prototype signal $g(t)$ defined as:

$$
T_{g}^{2}=4 \pi \int_{t} t^{2}|g(t)|^{2} d t, \quad F_{g}^{2}=4 \pi \int_{f} f^{2}|G(f)|^{2} d f,
$$

where $G(f)=\mathcal{F}\{g(t)\}$.

Under the assumptions (15), (16) or (17), and(18), one can show that the optimum scale of the prototype signal is characterized by the intuitively appealing rule:

$$
\frac{T_{g}}{F_{g}}=\frac{r_{\max }}{\nu_{\max }}
$$

this means that a rough matching may be obtained by starting with an arbitrary prototype function, holding the shape constant and optimizing its scale according to (20).

Matched Gaussian. For general elliptical symmetry of a given target function $T(\tau, \nu)=G\left(\left(\frac{\tau}{r_{a}}\right)^{2}+\left(\frac{\nu}{\nu_{0}}\right)^{2}\right)$ and $\alpha=0$ one can obtain exact analytic solutions of the prototype signal optimization criteria (13) and (14). Consistent with the above discussed approximate solutions, we find the Gaussian pulse with matched scale as a local optimum (other local optimas are the equally scaled Hermite functions which are no admissible solutions for most applications):

$$
g_{o p t}(t)=\tilde{g}_{o p t}^{(0)}(t)=\sqrt[4]{2 F_{g} / T_{g}} e^{-\pi\left(F_{g} / T_{g}\right) t^{2}},
$$

where $T_{g}$ and $F_{g}$ are the spectral and temporal moments defined according to (19). Here, the matching rule holds in a general form:

$$
\frac{T_{g}}{F_{g}}=\frac{\tau_{0}}{\nu_{0}}
$$

\section{CONCLUSIONS}

The WH structure is a natural choice given an incomplete a priori knowledge on practically important nonstationary environments such as the (underspread) WSSUS channel or underspread processes. We have discussed the problem of matching the prototype signal to such environments in a way that the corresponding discrete or continuous signal sets achieve approximate diagonalization. In particular it has been shown that matching the STFT/Gabor analysis window to an underspread process is equivalent to matching a signal to an underspread WSSUS channel. The optimization problem can be formulated in terms of the (magnitude squared) ambiguity function of the signal and the given spreading constraint for the process (channel). An approximate matching of the prototype signal can be obtained by fixing the shape and optimizing the scale.

\section{References}

[1] F. Hlawatsch and G. F. Boudreaux-Bartels, "Linear and quadratic time-frequency signal representations," IEEE Signal Processing Magazine, April 1992, pp. 21-67.

[2] W. Kozek, "Optimally Karhunen-Loeve-like STFT Expansion of Nonstationary Processes," Proc. IEEE ICA SSP-93, Minneapolis, MN, April 1993, Vo. 4, pp. 428-431.

[3] I. Daubechies, "The Wavelet transform, time-frequency localication and signal analysis," IEEE Trans. Info. Theory, Vol. 36, No. 5, Sept. 1990, pp. 961-1005.

[4] R. Tolimieri and R. Orr, "Characterization of WeylHeisenberg frames via Poisson summation relationships," Proc. IEEE ICASSP 92, Vol. 4, pp. 277-280.

[5] W. Kozek, "Matched generalized Gabor expansion of nonstationary processes," Proc. IEEE Int. Conf. Signals, Systems, and Computers, Pacific Grove, CA, Nov. 1993, pp. 499-503.

[6] R. Steele, Mobile Radio Communications, Pentech Press, London, 1992.

[7] W. Kozek, "On the generalized Weyl correspondence and its application to time-frequency analysis of linear time-varying systems," Proc. IEEE Int. Symp. on TimeFrequency and Time-Scale Analysis, Victoria, Canada, Oct. 1992, pp. 167-170.

[8] K. A. Sostrand, "Mathematics of the time-varying channel," Proc. of NATO Adv. Study Inst. on Sign. Proc. Vol. 2, Enschede (The Netherlands), 1968.

[9] F. G. Folland, Harmonic Analysis in Phase Space, Princeton University Press, 1989.

[10] A. Papoulis, Signal Analysis, McGraw-Hill, New York, 1984.

[11] C. H. Wilcox, "The synthesis problem for radar ambiguity functions," Technical Summary Report, Mathematics Research Center, United States Army, University of Wisconsin, April 1960.

[12] S. M. Sussman, "Least-square synthesis of radar ambiguity functions," IRE Trans. Information Theory, pp. 246-254, April 1962.

[13] W. Kozek, F. Hlawatsch, H. Kirchauer and U. Trautwein "Correlative time-frequency analysis and classification of nonstationary random processes," this issue.

[14] W. Kozek, "Matched Weyl-Heisenberg Expansions of Nonstationary Environments," Ph.D. Thesis, University of Technology Vienna, under review. 